\title{
A Dyadic Approach to Managing Heart Failure With Confidence
}

\author{
Karen S. Lyons, PhD; Ercole Vellone, PhD, RN; Christopher S. Lee, PhD, RN, FAHA, FAAN; \\ Antonello Cocchieri, PhD, RN; Julie T. Bidwell, BSN, RN; Fabio D’Agostino, PhD, RN; \\ Shirin O. Hiatt, MPH, MS, RN; Rosaria Alvaro, MSN, RN; Raul Juarez Vela, PhD, RN; \\ Barbara Riegel, DNSc, RN, FAHA, FAAN
}

\begin{abstract}
Background: The majority of heart failure (HF) self-care research remains focused on patients, despite the important involvement of family caregivers. Although self-care confidence has been found to play an important role in the effectiveness of HF self-care management on patient outcomes, no known research has examined self-care confidence within a dyadic context. Objective: The purpose of this study was to identify individual and dyadic determinants of self-care confidence in HF care dyads. Methods: Multilevel modeling, which controls for the interdependent nature of dyadic data, was used to examine 329 Italian HF dyads (caregivers were either spouses or adult children). Results: Both patients and caregivers reported lower-than-adequate levels of confidence, with caregivers reporting slightly higher confidence than patients. Patient and caregiver levels of confidence were significantly associated with greater patient-reported relationship quality and better caregiver mental health. Patient confidence in self-care was significantly associated with patient female gender, nonspousal care dyads, poor caregiver physical health, and low care strain. Caregiver confidence to contribute to self-care was significantly associated with poor emotional quality of life in patients and greater perceived social support by caregivers.

Conclusions: Findings are supportive of the need for a dyadic perspective of HF self-care in practice and research as well as the importance of addressing the needs of both members of the dyad to maximize optimal outcomes for both.
\end{abstract}

KEY WORDS: caregiver mental health, confidence, dyads, heart failure, relationship quality

Celf-care is critical to the effective management of $\checkmark$ heart failure (HF) and is linked directly with patientoriented and clinical outcomes. ${ }^{1}$ Healthcare providers often rely upon family members (primarily spouses and adult children) to engage in the maintenance and management of the patient's HF, especially when the patient's cognitive or physical impairment is severe. ${ }^{2}$ Although we have begun to understand the impact of HF on family caregivers and their role in patient outcomes, ${ }^{3-5}$ the

Karen S. Lyons, PhD

Associate Professor, School of Nursing, Oregon Health \& Science University, Portland, OR, USA.

Ercole Vellone, PhD, RN

Research fellow, Department of Biomedicine and Prevention, University of Rome Tor Vergata, Italy.

Christopher S. Lee, PhD, RN, FAHA, FAAN

Associate Professor, School of Nursing, Oregon Health \& Science University, Portland.

Antonello Cocchieri, PhD, RN

Consultant, Nursing Service, Catholic University, Rome, Italy.

Julie T. Bidwell, BSN, RN

PhD Student, School of Nursing, Oregon Health \& Science University, Portland.

Fabio D'Agostino, PhD, RN

Research fellow, Department of Biomedicine and Prevention, University of Rome Tor Vergata, Italy. majority of HF research remains focused on the patient. There is some evidence that HF care dyads who share responsibility and take a collaborative approach to selfcare have better dyadic and patient outcomes ${ }^{6,7}$ and that high levels of caregiver confidence in the patient's ability to engage in self-care are associated with positive patient outcomes. ${ }^{5,8}$ However, without a concerted effort to focus on the HF dyad as the unit of analysis using appropriate methodologies, the way patients with HF and

Shirin 0. Hiatt, MPH, MS, RN

Research Associate, School of Nursing, Oregon Health \& Science University, Portland.

Rosaria Alvaro, MSN, RN

Associate Professor, Department of Biomedicine and Prevention, University of Rome Tor Vergata, Italy.

Raul Juarez Vela, PhD, RN

Research Fellow, Faculty of Health Sciences, University San Jorge, Zaragoza, Spain.

Barbara Riegel, DNSc, RN, FAHA, FAAN

Professor, School of Nursing, University of Pennsylvania, Philadelphia.

The parent study was supported by the Center of Excellence for Nursing Scholarship in Rome.

The authors have no conflicts of interest to disclose.

\section{Correspondence}

Karen S. Lyons, PhD, School of Nursing, Oregon Health \& Science University, 3455 SW US Veterans Rd, SN-ORD, Portland, OR 97239 (Lyonsk@ohsu.edu).

DOI: 10.1097/JCN.0000000000000234 
caregivers work together and impact one another will continue to be overlooked (ie, the relational aspect of self-care). ${ }^{7,9}$

The Riegel model of HF self-care ${ }^{10}$ purports a naturalistic decision-making process that encompasses (1) routine behaviors to maintain physiologic homeostasis (self-care maintenance) as well as (2) evaluation and response to symptoms when they occur (self-care management). The confidence to perform such self-care (ie, self-care confidence) has been theorized to moderate the effect of self-care maintenance and management on patient outcomes in $\mathrm{HF}^{10}$ and found to play an important role in the effectiveness of HF self-care management on patient outcomes. ${ }^{10-12}$ Thus, identifying the determinants of HF self-care confidence is one pivotal way to minimize adverse consequences of $\mathrm{HF}$.

Patient self-care confidence has been found to be higher in patients with more education, ${ }^{13}$ lower illness severity, ${ }^{5,13,14}$ a more recent diagnosis, ${ }^{13}$ fewer comorbidities, ${ }^{13}$ as well as better physical and mental health. ${ }^{5,11,14-16}$ Moreover, patient self-care confidence is higher when the caregiver has better mental health ${ }^{8}$ and when either the patient or caregiver rates the quality of their relationship as high. ${ }^{8,17}$ The determinants of HF caregiver self-care confidence are unknown. In non-HF illness contexts, however, caregiver confidence has been associated with caregiver strain as well as mental health and patient physical health. ${ }^{18,19}$

Given the importance of HF self-care confidence in achieving optimal outcomes, the familial context of HF self-care, ${ }^{20}$ and the high levels of strain and psychological distress among HF caregivers, ${ }^{4,21-23}$ there is a pressing need to focus on the HF dyad to identify modifiable factors that are associated with better outcomes for both the patient and the caregiver. To date, few studies in HF have focused on the dyad as the unit of analysis ${ }^{17,24-27}$ and even fewer have used methodologies that appropriately control for the interdependent nature of dyadic data. ${ }^{9,28}$ Accordingly, the purpose of this study was to identify individual and dyadic determinants of patient and caregiver HF self-care confidence using multilevel modeling. We hypothesized that patient-, caregiver-, and dyadic-level factors would be significant in explaining variability in self-care confidence of both patients with $\mathrm{HF}$ and their spouse or adult-child caregivers.

\section{Methods}

\section{Design, Sample, and Setting}

The present study was a secondary analysis of a crosssectional study of Italian patients with HF and their caregivers. ${ }^{29}$ A convenience sample of 1192 patients with HF was enrolled from outpatient centers across 28 Italian provinces. Patients with HF were included if they were 18 years or older, had a confirmed diagnosis of $\mathrm{HF}$ via echocardiographic and clinical evidence according to the diagnostic criteria specified by the European Society of Cardiology, ${ }^{30}$ and were considered stable (ie, no acute coronary event in the previous 3 months). Patients with overt dementia were excluded. Caregivers who accompanied the patient to enrollment and were designated by the patient with HF as the primary caregiver were also invited to participate. Patients and caregivers who were willing and eligible to participate provided written informed consent and completed the survey at the time of the outpatient visit. Data collection was overseen by nurses trained in the study protocol, who provided assistance to patients when needed. The patients and the caregivers completed the surveys separately.

A total of $382 \mathrm{HF}$ dyads had available data for the current analysis. Owing to the important role differences between spouse and adult-child caregivers, the present analysis purposely focused on the $329(86 \%)$ HF dyads that comprised a spouse or adult-child caregiver (excluded dyads consisted of other relatives, friends, and formal caregivers). The study was approved by the institutional review boards at each outpatient center where the recruitment took place. In addition, the current analysis was determined by the Oregon Health \& Science University Institutional Review Board to be exempt (owing to adequate de-identification of data).

\section{Measures}

\section{Patient and Caregiver Self-care Confidence}

Patient confidence was measured using the Italian version of the 6-item confidence subscale of the Self-Care of HF Index v.6.2. ${ }^{31}$ The patients rate their ability to engage effectively in self-care on a 4-point scale. Standardized scores are calculated (range, $0-100$ ), with higher scores indicating greater confidence in their ability to keep free of HF symptoms, follow the treatment advice they have been given, evaluate the importance of HF symptoms, recognize changes in their health if they occur, do something that will relieve HF symptoms, and evaluate how well a remedy works. The subscale has demonstrated good reliability and validity in this population. ${ }^{31}$ Caregiver confidence was measured using the 6 -item confidence subscale of the Caregiver Contribution to Self-care of Heart Failure Index, ${ }^{32}$ a measure derived from the SelfCare of HF Index. The caregivers rate their confidence in their ability to contribute to the patient's self-care. Standardized scores are calculated (range, 0-100), with higher scores indicating greater confidence in their ability to keep the patient free of HF symptoms, follow the given treatment advice, evaluate the importance of symptoms, recognize changes in the patient's health when they occur, do something that will relieve the patient's symptoms, and evaluate how well a remedy works. The subscale has demonstrated good reliability and validity in the Italian population. $^{32}$ 


\section{Patient Cognitive Impairment}

Patient cognitive impairment was measured using the MiniMental State Examination (MMSE). ${ }^{33}$ The MMSE assesses orientation to time, attention and calculation, delayed recall, and language and visual construction. Scores on the MMSE range from 0 to 30, with lower scores indicating more cognitive impairment. The MMSE is the most widely used measure of mental status with good psychometric properties. ${ }^{34}$

\section{Patient and Caregiver Physical and Mental Health}

Patient physical and mental quality of life were measured using subscales from the 21-item Minnesota Living with Heart Failure Questionnaire. ${ }^{35}$ The Minnesota Living with Heart Failure Questionnaire is a condition-specific instrument; item responses range from 0 (none) to 5 (very much). Higher scores indicate worse physical and emotional quality of life. The measure has demonstrated strong reliability. ${ }^{36}$ Cronbach $\alpha$ in the present study was 0.89 . Caregiver physical and mental health were measured using the 2 subscales from the SF- 12 health survey. ${ }^{37}$ Scores on each component are standardized to range from 0 to 100 , with higher scores indicating better health. The SF-12 has demonstrated good reliability and validity in this population, ${ }^{38}$ including the present sample $(\alpha=0.85)$.

\section{Relationship Quality}

The patients and the caregivers were each asked to rate their relationship using single items. The patients rated the overall quality of the relationship on a scale of 0 (worst) to 5 (best) using an item created for the purpose of the parent study; the caregivers rated the overall quality of the relationship on a scale of 1 (never good) to 4 (always good) using an item from the Carers of Older People in Europe (COPE) Index. ${ }^{39}$

\section{Care Strain}

Caregiver strain was measured using the 24-item Caregiver Burden Inventory. ${ }^{40}$ The multidimensional measure is used to evaluate the impact of providing care on various aspects of the caregiver's life using a 5-point scale from 0 (not at all disruptive) to 4 (very disruptive). Higher scores indicate greater strain from providing care. The total score was used in the present study. The Cronbach $\alpha$ in the present study was 0.97 .

\section{Perceived Social Support}

Caregiver perceived social support was measured using the 4 -item subscale from the COPE Index. ${ }^{39}$ The caregivers were asked to rate the quality of social support they received on a scale of 1 (never) to 4 (always). Higher scores indicate greater perceived support. The COPE Index has been validated in several European languages, including Italian, and has demonstrated good reliability and validity. ${ }^{39,41}$

\section{Sociodemographic and Clinical Characteristics}

Patient and caregiver demographics were obtained via a self-reported survey (eg, age, gender, education, marital status, employment). In addition, patient clinical information was obtained from medical records (eg, duration of HF, hospitalization for HF within the last year, and the New York Heart Association functional class).

\section{Data Analyses}

Hierarchical linear modeling 7 (Skokie, Illinois) was used to analyze data at the level of the dyad to control for interdependencies in the data. ${ }^{42,43}$ The multivariateoutcome model is used to estimate a latent score for each member of the dyad (ie, 1 for the patient and 1 for the caregiver), controlling for the dependent nature of dyadic data. Such approaches are far superior to traditional methods and allow for direct examination of both actor (own) and cross-partner (other member of the dyad) effects. ${ }^{43,44}$ The level 1 (within-dyad) model represents the confidence scores $(Y)$ for both patient and caregiver as the sum of a latent true score $\left(\beta_{1}\right.$ for the patient and $\beta_{2}$ for the caregiver) plus a residual term $r$ that captures measurement error and was specified as follows:

$$
\mathrm{Y}_{\mathrm{ij}}=\beta_{1 \mathrm{j}}\left(\text { PATIENT }_{\mathrm{ij}}\right)+\beta_{2 \mathrm{j}}\left(\text { CAREGIVER }_{\mathrm{ij}}\right)+\mathrm{r}_{\mathrm{ij}}
$$

where $\mathrm{Y}_{\mathrm{ij}}$ represents the confidence score $i$ in dyad $j$ $(i=1, \ldots . . \mathrm{k}$ responses per dyad). PATIENT is an indicator variable taking on a value of 1 if the response was obtained from a patient and 0 if the response was obtained from a caregiver. CAREGIVER is an indicator variable taking on a value of 1 if the response was obtained from a caregiver and 0 if the response was obtained from a patient. Thus, $\beta_{1 \mathrm{j}}$ and $\beta_{2 \mathrm{j}}$ represent the patient's and caregiver's latent confidence scores, respectively. These estimates are known as fixed effects in the model. The $r_{i j}$ are the within-dyad residuals, also known as the level 1 random effects. The variance components associated with these random effects can be tested using a $\chi^{2}$ test to determine whether there is significant variability around average levels of patient and caregiver confidence. If there is, significant heterogeneity in confidence exists across dyads and independent variables can be included to explain this heterogeneity.

The level 2 (between-dyad) model consists of simultaneous regression equations with $\beta_{1 \mathrm{j}}$ and $\beta_{2 \mathrm{j}}$ now serving as dependent variables and can be specified as follows:

$$
\begin{aligned}
& \beta_{1 \mathrm{j}}=\gamma_{10}+\left[\gamma_{11} \text { Predictor }_{1}+\gamma_{1 \mathrm{n}} \text { Predictor }_{\mathrm{n}}\right] .+u_{1 \mathrm{j}} \\
& \beta_{2 \mathrm{j}}=\gamma_{20}+\left[\gamma_{21} \text { Predictor }_{1}+\gamma_{2 \mathrm{n}} \text { Predictor }_{\mathrm{n}}\right] .+u_{2 \mathrm{j}}
\end{aligned}
$$

$\gamma_{10}$ and $\gamma_{20}$ are the level 2 intercepts, representing average values of confidence for patient and caregiver, 
respectively, adjusted for the effects of the predictors in each equation. The fixed effects of each predictor are captured by the respective regression coefficient $\gamma$ and represent the association of each predictor with the confidence of either patients or caregivers. Taken together, these equations can account for patient and caregiver levels of confidence as a function of both individual and dyad-level factors.

A level 2 (between-dyad) model including both actor (eg, patient age predicting patient confidence; caregiver strain predicting caregiver confidence) and partner effects (eg, patient's cognitive impairment predicting caregiver's confidence; caregiver health predicting patient confidence) was examined. Independent variables were mean centered for ease of interpretation, except where a raw value of zero was considered meaningful (eg, number of hospitalizations). Coefficients for each independent variable are interpreted as unstandardized $B$ coefficients in a simultaneous multiple regression. A parallel scales approach was used to maximize reliability of the data in estimating level 1 models. ${ }^{45}$ Given the small number of items on the confidence subscale, each item served as a parallel scale, an approach considered more reliable than the alternative known variance method. ${ }^{42}$

\section{Results}

\section{Sample Characteristics}

The sample characteristics ( $\mathrm{n}=329 \mathrm{HF}$ dyads) are shown in Table 1 . The mean (SD) age of patients and their

TABLE 1 Sample Characteristics (N = 329 HF Dyads)

\begin{tabular}{|c|c|c|}
\hline & \multicolumn{2}{|c|}{$\begin{array}{l}\text { Mean } \pm \text { Standard } \\
\text { Deviation or } \mathbf{n}(\%)\end{array}$} \\
\hline & Patients & Caregivers \\
\hline Age, y & $76.8 \pm 9.7$ & $58.3 \pm 14.3$ \\
\hline Gender (\% female) & $144(44)$ & $179(54)$ \\
\hline Married & $204(62)$ & $258(78)$ \\
\hline Currently employed (\%) & $29(9)$ & $167(51)$ \\
\hline Education (\% high school or beyond) & $86(26)$ & $184(56)$ \\
\hline Caregiver type (\% spouse) & - & $133(40)$ \\
\hline \multicolumn{3}{|l|}{ NYHA class } \\
\hline |/II & $187(57)$ & - \\
\hline III/IV & $140(43)$ & - \\
\hline Months with HF & $58.7 \pm 47.6$ & - \\
\hline Hospitalized for HF in last year & $176(54)$ & - \\
\hline Cognitive impairment (MMSE score) & $23.9 \pm 6.3$ & - \\
\hline HF-specific physical quality of life ${ }^{a}$ & $22.0 \pm 8.4$ & - \\
\hline HF-specific emotional quality of life $e^{a}$ & $11.3 \pm 5.5$ & - \\
\hline General physical health ${ }^{\mathrm{b}}$ & - & $45.5 \pm 8.8$ \\
\hline General mental health ${ }^{\mathrm{b}}$ & - & $47.5 \pm 9.8$ \\
\hline Care strain & - & $29.9 \pm 23.9$ \\
\hline Perceived social support & - & $10.6 \pm 2.4$ \\
\hline Perceived relationship quality & $4.2 \pm 1.1$ & $3.2 \pm 0.9$ \\
\hline
\end{tabular}

NYHA, New York Heart Association.

${ }^{a}$ Minnesota Living with Heart Failure Questionnaire.

bSF-12 health survey. caregivers was 76.8 (9.7) and 58.3 (14.3) years, respectively. The patients were predominantly married $(62 \%)$ as well as cared for by an adult child $(60 \%)$, and just more than half the patient sample was male $(56 \%)$. The patients had been living with HF, on average, for almost 6 years; more than half of the patients had a New York Heart Association class I/II HF. Slightly more than half of the caregivers were women (54\%), and just more than half reported being currently employed. On average, the patients and the caregivers rated their relationship with each other as good.

\section{Dyadic HF Confidence}

It was shown in the results of the level 1 (within-dyad) model that the patients and the caregivers reported moderate levels of HF self-care confidence, with the caregivers reporting more confidence than the patients did (Table 2). More importantly, there was significant variability around the average scores for both the patients and the caregivers indicating significant heterogeneity in confidence across the dyads.

Patient- and caregiver-level factors that accounted for significant variability in HF self-care confidence across the HF dyads are presented in Table 3. Patient and caregiver levels of confidence were significantly higher when the patient rated the quality of the relationship higher, the caregiver experienced greater mental health, and the patient had lower levels of cognitive impairment. Certain factors only influenced the HF self-care confidence of the patients; patient female gender, nonspousal care dyads, poor caregiver physical health, and low care strain were significantly associated with higher levels of confidence in the patients. Other factors only influenced the self-care confidence of the HF caregiver; low patient education, worse HF-specific emotional quality of life, high caregiver education, and greater perceived social support by the caregiver were significantly associated with higher levels of confidence in caregivers.

\section{Discussion}

Little is known about how patients with HF and caregivers work together, and even less is known about the factors that influence their levels of confidence in contributing to HF self-care. The current study is the first study to examine the determinants of confidence in HF dyads using multilevel modeling, and several of our findings are noteworthy. First, most dyads reported moderate levels of confidence that fall short of recommended guidelines for adequate self-care. Second, the caregivers reported higher levels of self-care confidence than the patients with HF did. Third, there was considerable variability across the dyads in levels of HF self-care confidence reported by the patients and the caregivers. Finally, patient-, caregiver-, and dyadic-level factors all played 


\begin{tabular}{|c|c|c|c|}
\hline & Unstandardized $\beta$ & \multicolumn{2}{|c|}{$\boldsymbol{P}$} \\
\hline \multicolumn{4}{|l|}{ Fixed effects } \\
\hline Patient confidence & 50.81 & & \\
\hline Caregiver confidence & 53.79 & & \\
\hline Random effects & Variance Components & $\chi^{2}$ & $P$ \\
\hline Patient confidence & 193.40 & 744.06 & $<.001$ \\
\hline Caregiver confidence & 196.46 & 759.24 & $<.001$ \\
\hline
\end{tabular}

important roles in explaining variability in self-care confidence of both the patients with HF and their caregivers.

\section{Self-care Confidence of the Patients and the Caregivers}

Our finding that the patient's perception of the quality of the relationship with the caregiver was significantly associated with both the patient's and caregiver's level of confidence is consistent with both conceptualizations of confidence ${ }^{46}$ and previous research in $\mathrm{HF}^{8,12,17}$ Indeed, Bandura ${ }^{47}$ believed the illness process to be a social, not an individual one, with positive interactions with family members heightening confidence in managing chronic illness. In addition, the protective nature of relationship quality for HF caregivers has previously been demonstrated. ${ }^{6,12,48}$ Positive care relationships tend to encompass open communication and shared appraisals of symptoms, allowing for greater collaboration and shared decision making vital to achieving positive outcomes for both members of the dyad. ${ }^{20,49}$ Given the preponderance of protective buffering (ie, symptom concealment, communication avoidance) that accompanies chronic illness, ${ }^{49}$ interventions that directly facilitate communication and disclosure within care dyads and provide strategies to foster collaboration (eg, we-talk) are needed. The fact that the caregiver's perception of the quality of the relationship was not a significant determinant may be partly caused by the difference in items used for each member of the dyad, although the importance of the patient's perception of the relationship has also been found in chronic pain dyads. ${ }^{50}$

Both the patients and the caregivers reported lower self-care confidence when the caregiver experienced poor mental health. The negative consequences of providing care to a family member have been well documented $^{4,23,28,51}$ and are likely caused by the heavy symptom burden of HF and the emotional toll of caring for a family member with a terminal illness. Poor mental health impacts caregiver appraisals ${ }^{50,52}$ and may diminish the caregiver's capacity to be supportive. Evidence is also suggestive of the fact that caregiving interventions may be less effective in caregivers with high levels of depressive symptoms, ${ }^{53,54}$ indicating that caregiver mental health must be prioritized before expecting new skills. Not surprisingly, both the patients and the caregivers reported less confidence when the patient experienced cognitive impairment, reflecting the complexity of selfcare in the context of $\mathrm{HF}^{2}$

\section{Self-care Confidence of the Patients}

In contrast to previous research, we observed that men with HF reported lower levels of confidence than women did. ${ }^{13}$ Further work is needed to understand whether this is particular to the Italian sample or would be

\section{TABLE 3 Multilevel Model Level 2 Results Predicting Patient and Caregiver Confidence (N = 329 Dyads)}

\begin{tabular}{|c|c|c|c|c|}
\hline & \multicolumn{2}{|c|}{ Patients } & \multicolumn{2}{|c|}{ Caregivers } \\
\hline & Unstandardized $\beta$ & $P$ & Unstandardized $\beta$ & $P$ \\
\hline Intercept & 54.73 & $<.001$ & 53.29 & $<.001$ \\
\hline \multicolumn{5}{|l|}{ Patient variables } \\
\hline Age & -0.20 & .097 & -0.14 & .236 \\
\hline Gender (Male) & -5.15 & .010 & 0.80 & .696 \\
\hline Education (High school or beyond) & -2.79 & .314 & -7.25 & .007 \\
\hline Cognitive impairment (MMSE score) & 0.62 & $<.001$ & 0.52 & .008 \\
\hline HF-specific physical quality of life ${ }^{a}$ & -0.06 & .639 & 0.08 & .552 \\
\hline HF-specific emotional quality of life ${ }^{a}$ & 0.36 & .113 & 0.46 & .046 \\
\hline Hospitalized for HF last year & -0.88 & .452 & 0.43 & .721 \\
\hline Perceived relationship quality & 2.58 & .017 & 2.96 & $<.001$ \\
\hline \multicolumn{5}{|l|}{ Caregiver variables } \\
\hline Gender (female) & 1.09 & .562 & -0.15 & .940 \\
\hline Caregiver type (spouse) & -4.24 & .047 & -3.02 & .181 \\
\hline Education (high school or beyond) & 2.66 & .247 & 5.27 & .029 \\
\hline General physical health ${ }^{b}$ & -0.43 & .002 & -0.07 & .605 \\
\hline General mental health ${ }^{\mathrm{b}}$ & 0.28 & .022 & 0.43 & $<.001$ \\
\hline Care strain & -0.11 & .041 & 0.04 & .453 \\
\hline Relationship quality & 1.35 & .255 & 0.92 & .468 \\
\hline Perceived social support & 0.67 & .139 & 1.24 & .008 \\
\hline
\end{tabular}

Coefficients are adjusted for the influence of all multivariate model factors.

a Minnesota Living with Heart Failure Questionnaire.

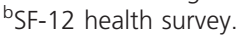


replicated in studies with more gender-balanced samples as was the case in the current study. Our results were also indicative that patients may be particularly at risk for low levels of self-care confidence when cared for by a spouse. Spouse caregivers in the current sample had significantly poorer physical health than the adult-child caregivers did. Thus, spousal caregivers may be managing their own illness and also may be less capable of engaging in HF self-care or feeling confident to do so. Patients with HF in these couples may need to be primarily responsible for the maintenance and management of their own illness and subsequently acquire greater expertise and confidence. This may also explain the association between high patient confidence and worse caregiver physical health. An interesting observation from these data show that, although more than $60 \%$ of the patients are married, only $40 \%$ are cared for by a spouse, suggesting that some adult children may be caring for 2 parents with illness. Thus, it is highly likely that this sample contained a subsample of couples with chronic illness where both spouses were in need of care. Alternatively, patients who have access to a caregiver with good physical health (more likely an adult child of the patient) may have less need to be engaged and confident in their own self-care.

Another important risk factor for patients is the level of strain felt by their caregiver. Such adverse consequences of providing care are known to lead to poor physical and mental health ${ }^{4,55,56}$ and, in the case of spouse caregivers, increased mortality. ${ }^{57}$ Thus, interventions that include components that address caregiver strain may be more effective in ameliorating both caregiver and patient outcomes and maximize the potential for caregivers to provide greater support. Finally, in contrast to prior research on HF self-care, ${ }^{6,11,23-26}$ we did not find patient education or quality of life to be significant determinants of patients' self-care confidence. It may be that the level of education and quality of life play less of a role in self-care confidence when more comprehensive multivariate models of patient and caregiver characteristics are examined.

\section{Self-care Confidence of the Caregivers}

Although patient level of education and health did not significantly predict patient confidence, as in previous research, caregiver confidence was lower when the patients reported good mental health and higher levels of education. Such patients may be more independent and engaged in their self-care, resulting in caregivers perceiving low need to become as engaged. Enactive engagement or "the doing" of a task has often been considered one of the strongest ways to increase levels of confidence $^{46}$; thus, caregivers with inadequate exposure or perceived opportunity to engage in HF self-care may be more likely to lack confidence to do so. Similar to the protective role of relationship quality (discussed previously), caregivers who reported high levels of social support experienced significantly higher confidence. This finding, once again, emphasizes the importance of acknowledging the relational and familial aspects of HF self-care for both patient and caregiver. Finally, as expected, caregivers with higher levels of education reported significantly more confidence.

\section{Strengths and Limitations}

The current study has several limitations. First, the crosssectional design of the study limits discussion of directional effects. Clearly, the cross-sectional data are suggestive that low confidence may lead to poor mental health, for example, as has been suggested by others. In reality, the relationship between these variables is most likely recursive, but without studies that explicitly examine dyads over time, we are not able to uncover directionality and more complex relationships. Second, it is unclear how much the current results will generalize beyond the Italian population. For example, caregiver gender was more balanced in the present study than the majority of studies reporting high percentages of female caregivers. When adult children become involved, older adults in Italy prefer to be cared for by someone of the same gender. In addition, the current study focused on spouse and adultchild caregivers. Thus, these findings will need to be replicated in other cohorts of patients with HF and their informal caregivers. Third, the patient sample was relatively healthy and there was large variability in levels of care strain experienced. It is unclear whether findings would be replicated in samples of predominantly advanced HF. Finally, our comprehensive models accounted for a moderate amount of variance in patient and caregiver self-care confidence, but there is clearly much more to be explained. Future research should include multiitem specific measures that capture varying aspects of collaboration and relationship quality (eg, communication, decision making, reciprocity, dyadic coping) that will be informative in leading to tailored interventions.

\section{Implications for Practice and Research}

Despite these limitations, the current study has several important strengths and implications for practice and research. First, this is one of the only known studies to examine modifiable determinants of confidence in contributing to self-care in HF dyads. By including both patient and caregiver using appropriate dyadic methodologies, we gain a more realistic estimate of the determinants of confidence to self-care for patients and their primary caregivers as well as potential avenues for intervention. In addition, the inclusion of both patient and caregiver predictor variables allowed us to examine both actor and partner effects to further understand the relational aspect of $\mathrm{HF}$ self-care. We found 4 partner effects for the patient and 3 partner effects for the caregiver, greatly reinforcing the need to take a dyadic approach to the study of HF. Second, the current study involved a more comprehensive predictive model than what was 


\section{What's New and Important}

Most HF care dyads reported moderate levels of confidence that fall short of recommended guidelines for adequate self-care.

- Patient-, caregiver-, and dyadic-level factors all played important roles in understanding dyadic confidence to self-care underscoring the relational aspect of HF.

- Taking a dyadic perspective to HF practice will allow clinicians to recognize care dyads with low levels of collaboration and communication and caregivers who may be experiencing high levels of depressive symptoms and strain.

previously examined with traditional demographic and individual health variables and also the inclusion of variables beyond the individual (ie, relationship quality, social support). The importance of these relational and social factors, controlling for individual variables, in culmination with the partner effects, underlines the need for health care providers to focus strategies and interventions at the level of the HF dyad, not the patient.

Taking a dyadic perspective to HF practice will allow clinicians to be vigilant for patients who may not be in a supportive care relationship while also identifying caregivers who may be experiencing high levels of depressive symptoms and strain as well as low levels of social support. Such modifiable risk factors are often closely intertwined. By facilitating conversations with the patient and the caregiver regarding their respective fears, concerns, and competing needs, clinicians can optimize the collaborative nature of the dyad, leading to increased confidence to self-care. Similarly, providing the dyad with problem-solving skills and fostering a team-based approach can assist the dyad in seeking out additional resources and social support needed to maintain the health and well-being of both patient and caregiver and, in some cases, the viability of the care dyad. In particular, clinicians need to be mindful of the emotional toll of the illness on both patient and caregiver with regular assessment of the caregiver recommended. Addressing the mental health needs of both members may lead to far more efficacious outcomes than those of traditional individual-level approaches. Cardiovascular nurses are ideally positioned to take a more dyadic perspective, identify at-risk care dyads, as well as provide needed resources and strategies. In addition, the cardiovascular nurse can work with families early in the HF trajectory (even before a caregiver perceives a need to actively engage) to help build caregiver confidence and more collaborative strategies so that both members of the dyad are better prepared when need increases.

\section{Conclusions}

Consistent with other research, levels of confidence to contribute to HF self-care fell far short of acceptable levels in the current study. Better caregiver mental health and greater relationship quality were the modifiable hallmarks of better self-care confidence in both the patient and the caregiver. More work is clearly needed, beyond traditional individual-level educational interventions, to increase confidence within HF dyads, given the importance of this concept to optimal patient outcomes and the clear social/relational aspect of HF. Targeting interventions and practice at the level of the HF dyad will lead to greater optimal outcomes for both patient and caregiver.

\section{REFERENCES}

1. Riegel B, Moser DK, Anker SD, et al. State of the science: promoting self-care in persons with heart failure: a scientific statement from the American Heart Association. Circulation. 2009;120:1141-1163.

2. Lee CS, Gelow JM, Bidwell JT, et al. Blunted responses to heart failure symptoms in adults with mild cognitive dysfunction. J Cardiovasc Nurs. 2013;28:534-540.

3. Buck HG, Harkness K, Wion R, et al. Caregivers' contributions to heart failure self-care: a systematic review [published online ahead of print]. Eur J Cardiovasc Nurs. 2014.

4. Chung ML, Lennie TA, Mudd-Martin G, Dunbar SB, Pressler SJ, Moser DK. Depressive symptoms in patients with heart failure negatively affect family caregiver outcomes and quality of life [published online ahead of print]. Eur J Cardiovasc Nurs. 2014.

5. Rohrbaugh MJ, Shoham V, Coyne JC, Cranford JA, Sonnega JS, Nicklas JM. Beyond the "self" in self-efficacy: spouse confidence predicts patient survival following heart failure. J Fam Psychol. 2004;18:184-193.

6. Rohrbaugh MJ, Mehl MR, Shoham V, Reilly ES, Ewy GA. Prognostic significance of spouse we talk in couples coping with heart failure. J Consult Clin Psychol. 2008;76:781-789.

7. Sebern M, Woda A. Shared care dyadic intervention: outcome patterns for heart failure care partners. West J Nurs Res. 2012; 34:289-316.

8. Benazon NR, Foster MD, Coyne JC. Expressed emotion, adaptation, and patient survival among couples coping with chronic heart failure. J Fam Psychol. 2006;20:328-334.

9. Vellone E, Chung ML, Cocchieri A, Rocco G, Alvaro R, Riegel B. Effects of self-care on quality of life in adults with heart failure and their spousal caregivers: testing dyadic dynamics using the actorpartner interdependence model. J Fam Nurs. 2014;20:120-141.

10. Riegel B, Dickson VV. A situation-specific theory of heart failure self-care. J Cardiovasc Nurs. 2008;23:190-196.

11. Lee CS, Suwanno J, Riegel B. The relationship between self-care and health status domains in Thai patients with heart failure. Eur J Cardiovasc Nurs. 2009;8:259-266.

12. Sayler J, Schubert CM, Chiaranai C. Supportive relationships, self-care confidence, and heart failure self-care. J Cardiovasc Nurs. 2012;27:384-393.

13. Lee CS, Riegel B, Driscoll A, et al. Gender differences in heart failure self-care: a multinational cross-sectional study. Int J Nurs Stud. 2009;46:1485-1495.

14. Arnold R, Ranchor AV, DeJongste MJL, et al. The relationship between self-efficacy and self-reported physical functioning in chronic obstructive pulmonary disease and chronic heart failure. Behav Med. 2005;31:107-115.

15. Goodman H, Firouzi A, Banya W, Lau-Walker M, Cowie MR. Illness perception, self-care behaviour and quality of life of heart failure patients: a longitudinal questionnaire survey. Int J Nurs Stud. 2013;50:945-953.

16. Buck HG, Lee CS, Moser DK, et al. Relationship between selfcare and health-related quality of life in older adults with moderate to advanced heart failure. J Cardiovasc Nurs. 2012;27:8-15. 
17. Sebern M, Riegel B. Contributions of supportive relationships to heart failure self-care. Eur J Cardiovasc Nurs. 2009;8:97-104.

18. Keefe FJ, Ahles TA, Porter LS, et al. The self-efficacy of family caregivers for helping cancer patients manage pain at endof-life. Pain. 2003;103:157-162.

19. Porter LS, Keefe FJ, Garst J, McBride CM, Baucom D. Selfefficacy for managing pain, symptoms, and function in patients with lung cancer and their informal caregivers: associations with symptoms and distress. Pain. 2008;137:306-315.

20. Dunbar SB, Clark PC, Quinn C, Gary RA, Kaslow NJ. Family influences on heart failure self-care and outcomes. J Cardiovasc Nurs. 2008;23:258-265.

21. Martensson J, Dracup K, Canary C, Fridlund B. Living with heart failure: depression and quality of life in patients and spouses. J Heart Lung Transplant. 2003;22:460-467.

22. Pihl E, Jacobsson A, Fridlund B, Stromberg A, Martensson J. Depression and health-related quality of life in elderly patients suffering from heart failure and their spouses: a comparative study. Eur J Heart Fail. 2005;7:583-589.

23. Trivedi RB, Piette J, Fihn SD, Edelman D. Examining the interrelatedness of patient and spousal stress in heart failure: conceptual model and pilot data. J Cardiovasc Nurs. 2012;27:24-32.

24. Agren S, Evangelista LS, Hjelm C, Stromberg A. Dyads affected by chronic heart failure: a randomized study evaluating effects of education and psychosocial support to patients with heart failure and their partners. J Card Fail. 2012;18:359-366.

25. Buck HG, Zambroski CH, Hupcey JE. Assessing the response of patient and spousal/partner caregivers to a new instrument measuring dyadic heart failure care types. Eur J Cardiovasc Nurs. 2013;12:209-213.

26. Bekelman DB, Nowels CT, Retrum JH, et al. Giving voice to patients' and family caregivers' needs in chronic heart failure: implications for palliative care programs. J Palliat Med. 2011; 14:1317-1324.

27. Luttik ML, Leegte IL, Jaarsma T. Quality of life and depressive symptoms in heart failure patients and their partners: the impact of role and gender. J Card Fail. 2009;15:580-585.

28. Chung ML, Moser DK, Lennie TA, Rayens MK. The effects of depressive symptoms and anxiety on quality of life in patients with heart failure and their spouses: testing dyadic dynamics using actor-partner interdependence model. J Psychosom Res. 2009;67:29-35.

29. Cocchieri A, Riegel B, D'Agostino F, et al. Describing self-care in Italian adults with heart failure and identifying determinants of poor self-care [published online ahead of print]. Eur J Cardiovasc Nurs. 2014.

30. McMurray JJ, Adamopoulos S, Anker SD, et al. ESC guidelines for the diagnosis and treatment of acute and chronic heart failure 2012: The Task Force for the Diagnosis and Treatment of Acute and Chronic Heart Failure 2012 of the European Society of Cardiology. Developed in collaboration with the Heart Failure Association (HFA) of the ESC. Eur J Heart Fail. 2012;14(8):803-869.

31. Vellone E, Riegel B, Cocchieri A, et al. Psychometric testing of the Self-Care of Heart Failure Index Version 6.2. Res Nurs Health. 2013;36(5):500-511.

32. Vellone E, Riegel B, Cocchieri A, et al. Validity and reliability of the caregiver contribution to self-care of heart failure index. J Cardiovasc Nurs. 2013;28(3):245-255.

33. Folstein MF, Folstein SE, McHugh PR. "Mini-mental state". A practical method for grading the cognitive state of patients for the clinician. J Psychiatr Res. 1975;12(3):189-198.

34. Kane RL, Kane RA, eds. Assessing Older Persons: Measures, Meaning, and Practical Applications. New York, NY: Oxford University Press; 2000.

35. Rector TS, Kubo SH, Cohn JN. Validity of the Minnesota Living with Heart Failure questionnaire as a measure of ther- apeutic response to enalapril or placebo. Am J Cardiol. 1993; 71(12):1106-1107.

36. Rector T. Overview of The Minnesota Living with Heart Failure Questionnaire. http://www.mlhfq.org/. Last updated January 1, 2005. Accessed September 28, 2009.

37. Ware JJr, Kosinski M, Keller SD. A 12-Item Short-Form Health Survey: construction of scales and preliminary tests of reliability and validity. Med Care. 1996;34(3):220-233.

38. Gandek B, Ware JE, Aaronson NK, et al. Cross-validation of item selection and scoring for the SF-12 Health Survey in nine countries: results from the IQOLA project. International Quality of Life Assessment. J Clin Epidemiol. 1998;51:1171-1178.

39. McKee KJ, Philp I, Lamura G, et al. The COPE index - a first stage assessment of negative impact, positive value and quality of support of caregiving in informal carers of older people. Aging Ment Health. 2003;7(1):39-52.

40. Novak M, Guest C. Application of a multidimensional caregiver burden inventory. Gerontologist. 1989;29(6):798-803.

41. Balducci C, Mnich E, McKee KJ, et al. Negative impact and positive value in caregiving: validation of the COPE index in a six-country sample of carers. Gerontologist. 2008;48(3):276-286.

42. Barnett RC, Marshall NL, Raudenbush SW, Brennan RT. Gender and the relationship between job experiences and psychological distress: a study of dual-earner couples. J Pers Soc Psychol. 1993;64:794-806.

43. Sayer AG, Klute MM. Analyzing couples and families: multilevel methods. In: Bengtson VL, Acock AC, Allen KR, DilworthAnderson P, Klein DM, eds. Sourcebook on Family Theory and Research. Thousand Oaks, CA: Sage; 2005:289-313.

44. Kenny DA, Kashy DA, Cook WL. Dyadic Data Analysis. New York, NY: The Guildford Press; 2006.

45. Lyons KS, Sayer AG. Longitudinal dyad models in family research. J Marriage Fam. 2005;67:1048-1060.

46. Bandura A. Social Foundations of Thought and Action. Upper Saddle River, NJ: Prentice Hall; 1986.

47. Bandura A. Self-Efficacy: The Exercise of Control. New York, NY: Freeman; 1997.

48. Luttik ML, Blaauwbroek A, Dijker A, Jaarsma T. Living with heart failure: partner perspectives. J Cardiovasc Nurs. 2007; 22:131-137.

49. Berg CA, Upchurch R. A Developmental-Contextual model of couples coping with chronic illness across the adult life span. Psychol Bull. 2007;133:920-954.

50. Lyons KS, Jones KD, Bennett RM, Hiatt SO, Sayer AG. Couple perceptions of fibromyalgia symptoms: the role of communication. Pain. 2013;154:2417-2426.

51. Agren S, Evangelista LS, Stromberg A. Do partners of patients with heart failure experience caregiver burden? Eur J Cardiovasc Nurs. 2010;9:254-262.

52. Cano A, Johansen AB, Franz A. Multilevel analysis of couple congruence on pain, interference, and disability. Pain. 2005; 118:369-379.

53. Coon DW, Thompson L, Steffen A, Sorocco K, GallagherThompson D. Anger and depression management: psychoeducational skill training interventions for women caregivers of a relative with dementia. Gerontologist. 2003;43:678-689.

54. Northouse LL, Katapodi MC, Song L, Zhang L, Mood DW. Interventions with family caregivers of cancer patients: a metaanalysis of randomized trials. CA Cancer J Clin. 2010;60:317-339.

55. Pressler SJ, Gradus-Pizlo I, Chubinski SD, et al. Family caregivers of patients with heart failure: a longitudinal study. J Cardiovasc Nurs. 2013;28:417-428.

56. Pinquart M, Sorensen S. Differences between caregivers and noncaregivers in psychological health and physical health: a meta-analysis. Psychol Aging. 2003;18:250-267.

57. Schulz R, Beach SR. Caregiving as a risk factor for mortality: the caregiver health effects study. JAMA. 1999;282:2215-2219. 